Intelligent Sensor Tasking for Space Collision Mitigation

S. S. Olivier, A. J. Pertica, J. R. Henderson

April 8, 2010 
This document was prepared as an account of work sponsored by an agency of the United States government. Neither the United States government nor Lawrence Livermore National Security, LLC, nor any of their employees makes any warranty, expressed or implied, or assumes any legal liability or responsibility for the accuracy, completeness, or usefulness of any information, apparatus, product, or process disclosed, or represents that its use would not infringe privately owned rights. Reference herein to any specific commercial product, process, or service by trade name, trademark, manufacturer, or otherwise does not necessarily constitute or imply its endorsement, recommendation, or favoring by the United States government or Lawrence Livermore National Security, LLC. The views and opinions of authors expressed herein do not necessarily state or reflect those of the United States government or Lawrence Livermore National Security, LLC, and shall not be used for advertising or product endorsement purposes.

This work performed under the auspices of the U.S. Department of Energy by Lawrence Livermore National Laboratory under Contract DE-AC52-07NA27344. 


\title{
Intelligent sensor tasking for space collision mitigation
}

\author{
Scot S. Olivier*, Alexander J. Pertica, John R. Henderson, \\ Sergei Nikolaev, Don Phillion, Wim De Vries \\ Lawrence Livermore National Laboratory, 7000 East Avenue, Livermore CA, 94551-9234
}

\begin{abstract}
Orbital collisions pose a hazard to space operations. Using a high performance computer modeling and simulation environment for space situational awareness, we explore a new paradigm for improving satellite conjunction analysis by obtaining more precise orbital information only for those objects that pose a collision risk greater than a defined threshold to a specific set of satellites during a specified time interval. In particular, we assess the improvement in the quality of the conjunction analysis that can be achieved using a distributed network of ground-based telescopes.
\end{abstract}

Keywords: Space situational analysis, conjunction analysis, telescope network, high performance computing, modeling and simulation

\section{INTRODUCTION}

Conjunction analysis is used to predict how close two space objects will come to each other. In the case of an operational satellite and a second satellite or a piece of space debris, a critical question is whether the minimum separation results in an unacceptable probability of collision, and the operational satellite should be moved. In practice, there can be significant uncertainties in the predicted minimum separation when using the publicly available catalog of satellite position information, so a $10 \mathrm{~km}$ threshold is often used to generate a warning of a conjunction. This results in hundreds of conjunction warnings every day for the approximately 1300 operational satellites. The Joint Space Operations Center (JSpOC) uses the full information from the Space Surveillance Network, and estimates it will generate about 75 warnings per day for the operational satellites [1]. These warnings from the JSpOC resulted in 37 known debris avoidance maneuvers for commercial and foreign satellites in a 10 month period in 2009 [1]. For high value objects, such as the International Space Station, additional information is acquired to refine the orbits of the two objects and generate a higher quality collision probability to ensure the collision risk justifies the cost of an avoidance maneuver.

The problem addressed here is that the vast majority of current conjunction warnings have too large a position uncertainty to justify an avoidance maneuver, and that there is currently no readily-tasked set of telescopes that can be used to refine the orbital information and improve the collision probability estimate. When it is necessary to obtain better orbital information for conjunctions involving high value objects, assets outside the SSN are typically used because the SSN assets are typically fully scheduled for their SSN and other tasks and because that network is not set up for ad hoc tasking requests. We consider here the improvement in conjunction analysis that could be obtained by a distributed network of ground-based telescopes, where the tasking of this network is cued by the results of the conjunction analysis using information from the SSN. A significant factor in the uncertainty of the conjunction analysis is that orbital information goes "stale" due to the complexity of accurately propagating an orbit forward in time. The scheme described here combines the best features of both systems - tracking of all space objects by the SSN, and timely collection of orbital information by a taskable network of ground-based telescopes.

Lawrence Livermore National Laboratory (LLNL) has developed a suite of modeling, simulation and visualization tools to help transform the United State's abilities in Space Situational Awareness (SSA). The Testbed Environment for Space Situational Awareness (TESSA) is an integrated framework for the utilization of these tools. A paper [2] describing the initial implementation of TESSA was published in the proceedings of the September 2008 Advanced Maui Optical and Space Surveillance Technologies Conference.

*olivier1@1lnl.gov, phone 1-925-423-6483 
TESSA is based on a flexible, scalable architecture, exploiting LLNL high-performance computing capabilities, to enable efficient, physics-based simulation of the current SSA enterprise, and to accommodate future advancements in SSA systems. TESSA includes hydrodynamic models of satellite intercept and debris generation, orbital propagation algorithms, radar cross section calculations, optical brightness calculations, radar system models, optical system models, object detection algorithms, orbit determination algorithms, simulation analysis and visualization tools. By effectively linking these detailed modules together, using an efficient "parallel discrete event simulation" architecture, TESSA provides a fundamentally new capability for the country that can be used to drive development of game-changing advances in SSA through dramatic increases in effective utilization of existing data sources and coherent planning for new sensor systems tuned to specific threat scenarios.

Here we apply some of our TESSA capabilities, both within the TESSA framework and as separate entities, to conjunction analysis to show that intelligent tasking of a network of ground telescopes can provide the information needed to reduce the uncertainty in conjunction analysis sufficiently to result in actionable information about the collision probability.

\section{SPACE COLLISION MITIGATION METHODLOLGY}

\subsection{Summary}

A standard catalog of space objects was used, and a new object, "CollisionSat" (CS) was added. The orbit of CS was chosen to either miss or collide with the Cibola Flight Experiment (CFE) satellite. Conjunction analysis was performed looking out several days in the future. The close conjunction of CFE and CS was used to generate tasking for a network of ground-based telescopes. Observations from those telescopes were simulated, and the streaks in the simulated imagery used to refine the orbit of CFE. CS was assumed to have a well known position (e.g., from on-board GPS) and accurate orbit determination. After each observation of CFE, the conjunction analysis was repeated for those two satellites, and an updated collision probability generated.

We used both Monte Carlo and analytical methods to calculate the collision probability. One starts with the calculated satellite trajectory and uncertainty in position, and can generate the collision probability from the overlap of the probability distribution function for each satellite. A useful view of the problem is to consider all of the positional uncertainty to reside with one satellite, and all of the physical cross-sectional area to belong to the other. The collision probability is then just the integral of the probability density for the first satellite over the area of the second. If the spatial separation of the two satellites is greater than the width of the probability distribution, the overlap and the collision probability will be small. Reducing the uncertainty in the satellite positions will reduce the width of the probability distribution, and reduce the collision probability, assuming the spatial separation does not change. On the other hand, if there is a small (or no) separation between the satellites, reducing the width of the probability distribution will increase the probability density at the location of the second satellite, and the collision probability will increase.

Data plots were generated of the collision probability and the rms size of the positional error ellipsoid of each satellite. The latter is used to track the relative change in the uncertainty of the satellite position, but cannot be directly compared to that uncertainty because the largest uncertainty in the satellite position is in the along-orbit direction, whereas the collision probability depends on the full 3D geometry of the error ellipsoids as well as the timing of the conjunction. This raises the point that there can be two types of misses for a close conjunction. The first is a spatial miss, where the satellite orbits do not overlap. The second is a temporal miss, where the orbits do overlap, but the two objects do not occupy the overlap region at the same time. We investigated both types of misses.

\subsection{Conjunction Screening and Collision Probability}

We use the SGP4 propagator to rapidly scan the $\mathrm{M} \times \mathrm{N}$ potential conjunctions for ones that come closer than a certain threshold over the period under consideration. Given the limited intrinsic positional accuracy of the TLE catalog, we are using a rather conservative threshold of 10 kilometers. Depending on the size of the $\mathrm{M}$ and $\mathrm{N}$ catalogs, this usually reduces the subset of close conjunctions down to the level of a few hundred per day. 
On this reduced set we then run the full Force Model calculation (see Section 2.5). This allows us to have the Covariance Matrix and State Vector information for each of these objects at any given time, and usually leads to improved estimates of the time and nearest approach of their close conjunctions.

The collision probability between two orbiting objects is given by the volume integral of the combined probability density function of each object. The individual probability density functions of the objects are based on their covariance matrix at the time of closest approach, and can be pictured as three-dimensional ellipsoids that vary along their orbits. Since we are considering a typical short-term LEO encounter on nearly circular orbits, we assume that the uncertainty ellipsiods are constant for the duration of the encounter.

For each of the two objects involved in the collision probability calculation, we generate a large number of random positions (on the order of a million) based on the eigenvalues and eigenvectors of the Covariance Matrix. Call these sets $\mathrm{A}$ and $\mathrm{B}$, each containing $\mathrm{N}$ positions. We further assume that each random coordinate value is distributed in a Gaussian way about the unperturbed position, with the magnitudes of the sigmas set by the square root of the relevant eigenvalue. In most cases the off-diagonal terms of the Covariance matrix are non-zero, signifying strong correlations between the uncertainties. This is being taken care of by multiplying the random vectors by the relevant normalized eigenvectors. Both sets A and B of these random, correlated vectors are then rotated into a frame where the relative velocity vector aligns with the $\mathrm{z}$ axis. The plane perpendicular to this approach direction is called the collision plane.

The simplest Monte Carlo calculation of the collision probability is now defined as the number of distances in the $(\mathrm{x}, \mathrm{y})$ plane between points belonging to A and B that are smaller than the collision cross-section, divided by the total number of permutations considered. In other words, we are projecting all the random vectors onto the collision plane before we start correlating sets $\mathrm{A}$ and $\mathrm{B}$. It should also be noted that we are not using all $\mathrm{N} \mathrm{x} \mathrm{N}$ possible permutations as they do not form a truly random $\mathrm{N}^{\wedge} 2$ set, and that beyond our $\operatorname{sqrt}(\mathrm{N}) \mathrm{xN}$ number of select permutations no extra numerical fidelity is gained.

We also considered more refined Monte Carlo approaches where we go beyond a straight projection onto the collision plane, but for the scenarios under consideration in this paper, we found the simple projection technique to be adequate.

\subsection{Ground network and tasking}

The simulations are done using a network of ground-based binocular telescopes, placed randomly and uniformly around the globe. The first telescope in each pair is picked by choosing $\lambda$ randomly and uniformly from the interval $\left[-180^{\circ},+180^{\circ}\right]$ and by choosing $\sin (\varphi)$ randomly and uniformly from the interval $[-1,+1]$. The placement disregards whether it is on land or water. The probability density for placing the first telescope in each pair is uniform in $(\lambda, \varphi)$ and disregards whether the proposed site is on the ground or on the water. The altitude for the first site $\mathrm{h}_{1}$ is drawn uniformly between 0 and 3000 meters. Given the location of the first telescope in a pair, the location for the second telescope is defined by randomly orienting a $20 \mathrm{~km}$ baseline vector, and drawing an altitude value uniformly between $\mathrm{h}_{1}$ and $\mathrm{h}_{1}+100$ meters.

The scheduling for components of each binocular telescope is time-synchronized, i.e. exposures are always taken at the same MJD, for the same time interval. This is done to facilitate processing of the resulting satellite streaks and triangulating. The tasking of telescopes is effected by sending a schedule request (an XML packet) to the Scheduler module in TESSA, requesting observation of particular satellite within a given time interval. The request can be addressed to all telescopes in the simulation, or may stipulate a specific binocular telescope to use. The Scheduler calculates visibility of the satellite for each requested telescope on 10-sec time grid. The visibility conditions include checks for elevation of the satellite $\left(\mathrm{e}>\mathrm{e}_{\min }\right)$, Sun's elevation $\left(\mathrm{e}_{\mathrm{Sun}}<-15^{\circ}\right)$, and whether the satellite is in the Earth's shadow. Assuming all three conditions are satisfied, the pointing information $\left(\alpha, \delta, M J D, t_{\text {exp }}\right.$, filter $)$ is generated and added to a list. The lists of pointings for two telescopes in a pair can differ slightly because of the $20 \mathrm{~km}$ baseline between the instruments: even though the pointings are calculated on a synchronized time grid, some may be lost due to visibility constraints. To ensure complete synchronization, we take the intersection of pointings lists and send the resulting observation schedule to the telescopes.

\subsection{Optical telescope modeling}

Once a telescope receives an observation schedule, it proceeds to generate an "observed" image for each pointing. This is handled by the Optical Detection Pipeline module of TESSA, which consists of two parts: image generation and image analysis. In the image generation stage, the pipeline models a variety of effects, including atmospheric light scattering, 
stellar component (including unresolved stars), variable flux due to tumbling motions of orbital debris, light propagation through the telescope optics, tracking mode, charge transfer artifacts, weather conditions, various noise sources, etc. As a result, it produces a realistic-looking optical image of the star field and satellites (if any are in the field-of-view). The images, written as FITS (Flexible Image Transport System) files, are shipped to the next stage of the pipeline for processing.

During the processing stage, the raw pixel values in the image are analyzed to extract sources (stars and satellites). We found that elongated satellite streaks are best characterized using a Canny edge detector [3], which calculates the footprint of each object using the gradient map of the image. The footprints are analyzed to extract the endpoints of each streak in image coordinates. Combined with the knowledge of the WCS (World Coordinate System) of the image and the timing information, these endpoints can be converted into RA and Dec, and processed to extract orbital information. An example fragment of the image with a satellite streak, along with the extracted footprints is shown in Figure 1.

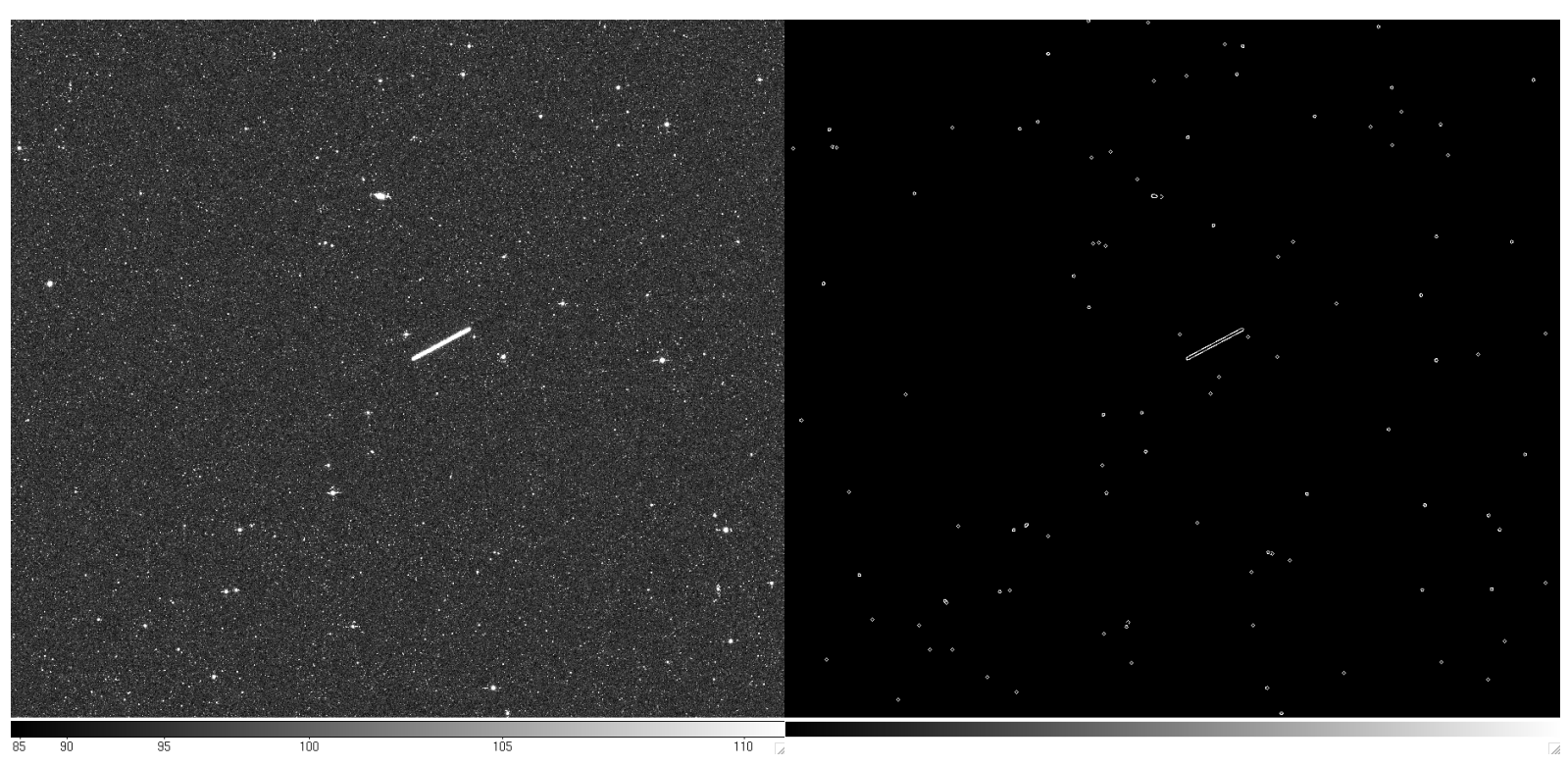

Figure 1. Fragment of the "observed" image of a satellite streak on a stellar background (left) and the corresponding extracted objects using Canny edge detector (right).

\subsection{Streak aggregation, orbit refinement or orbit determination, catalog updating, and conjunction probability determination}

Part of the TESSA architecture is depicted in the figure shown below. The output of the telescopes is an ImageStreaks XML file for each exposure. This ImageStreaks file has the endpoint RAt and DECt angles for all the streaks in the image. It also has the errors for the streaks. Even if there are no streaks for an exposure, an ImageStreaks file for that exposure is still created and sent to the streak aggregator. This behavior is required by the PDES framework. It is the job of the streak aggregator to make tracks from the streaks. There is a single telescope streak aggregator based upon class StreakAggregator and a more general multi-telescope streak aggregator which maintains a queue of class StreakAggregator objects. This multi-telescope streak aggregator can recognize paired streaks from binocular telescopes, and, for paired streaks from a catalog object, triangulate at the endpoints to obtain the ranges for the two telescopes. Since three-dimensional positions for the object have been obtained at the streak endpoint times, the streak that would be seen by any telescope for this exposure time and duration is known. For convenience, the streak that would be seen by a fictitious midway telescope is computed and we pretend that the streak was observed by this telescope. Streaks which have the correct streak endpoints for a catalog object within the errors are put into the track for that object for that telescope.

Streaks that do not associate to catalog objects are put together into tracks on the basis of several criteria. Each streak added to a track is required to have about the same direction as the preceding streak in that track. Furthermore, the ratio 
of the streak arc length to exposure time must be about the same as the ratio of the streak arc length to the exposure time for the previous streak. Lastly, the ratio of the streak arc length to the center-to-center distance between that streak and the previous streak must be about the same as the ratio of the exposure time to the time between exposures. Streaks that can't be assigned to an existing track are assigned to a new track.

A track is closed and written as an XML file if no new streak has been added to that track within the rather arbitrary time of twenty minutes. Each track is for a single pass of a single object seen by a single telescope except that for binocular telescopes the streaks are paired and represented as being observed from a fictitious midway telescope. These streaks have ranges as well as RAt and DECt angles at their endpoints.

The binocular telescope with triangulation to get the ranges at the streak endpoints results in a vastly superior initial orbit than does using only the line-of-sight data from a single telescope when using Herrick-Gibbs initial orbit determination for the binocular telescope data with ranges and using Laplace's method for the line-of-sight data. Laplace's method have been generalized to use all the observations in a track, not just three observations. Batch least squares orbit refinement would then be used following this initial orbit determination, but if the initial orbit determination is of too low a quality, it will diverge. Here is a comparison for one aggregated binocular telescope track of the initial orbit determination using either Herrick-Gibbs [4] or Laplace's [5] method:

$\begin{array}{rrrr} & \text { Herrrick-Gibbs } & \text { Laplace's } & \text { Actual } \\ \text { Mjd } & 55045.76111 & 55045.76111 & 55045.94505 \\ \mathrm{a} & 6925091 & 6598753 & 6936461 \\ \mathrm{e} & 0.00198919 & 0.05247634 & 0.0003904 \\ \mathrm{i} & 35.421194^{\circ} & 35.499233^{\circ} & 35.4317 \\ \Omega & 180.887170^{\circ} & 182.728148^{\circ} & 179.7644 \\ \omega & 323.955642^{\circ} & 280.120850^{\circ} & 293.9467 \\ \mathrm{M} & 119.098669^{\circ} & 159.830672^{\circ} & 66.0847 \\ \text { rms angle error } & 0.007 & 0.416 & \mathrm{~N} / \mathrm{A} \\ \text { rms position error } & 145.3 & 8050.7 & \mathrm{~N} / \mathrm{A}\end{array}$

The ODAA (Orbit Determination And Association) will do the first orbit determination and the batch least squares orbit refinement for tracks which have not been associated to catalog objects. The ODAA sends an ODAA report for every track to the MTA (Multiple Track Aggregation). This report includes the name of the track file. Each time there is a new track for a catalog object, the MTA uses all the tracks for that object to refine the catalog orbit using sequential least squares. The MTA uses an estimated initial catalog which can differ from the truth catalog used to generate the sky pictures for the telescopes and the radars.

The MTA keeps an updated catalog. This updated catalog includes both the updated orbits and the covariance matrices. Presently, the MTA does not associate uncorrelated tracks but this is on the agenda for future TESSSA development, and both we and the AMOS/MHPCC team have done work developing algorithms for associating uncorrelated tracks based upon the covariance matrix. Extended Kalman filters will also be added to the MTA in future work.

The ConMod component sends Conjunction Analysis Requests to the MTA asking for the updated orbits and covariance matrices for pairs of objects at their close conjunction times. In response, the MTA sends CollisionCheck XML files to the CPMod component. At each request time, for each object that CollisionCheck XML file has the updated state vectors and covariance matrices propagated to the conjunction time. For each close conjunction, the CPMod component computes the collision probability given the object sizes using the Chan analytic method, the Foster (NASA) analytic method, and the Monte Carlo method.

On the agenda for future TESSA development is the force model using a force model table for speed. A simplified TESSA using the force model table was successfully implemented last summer. This simplified TESSA included only the Radar and ODAA components. So far, all the TESSA components except for the Telescopes and the MTA have been made "force model compliant". 


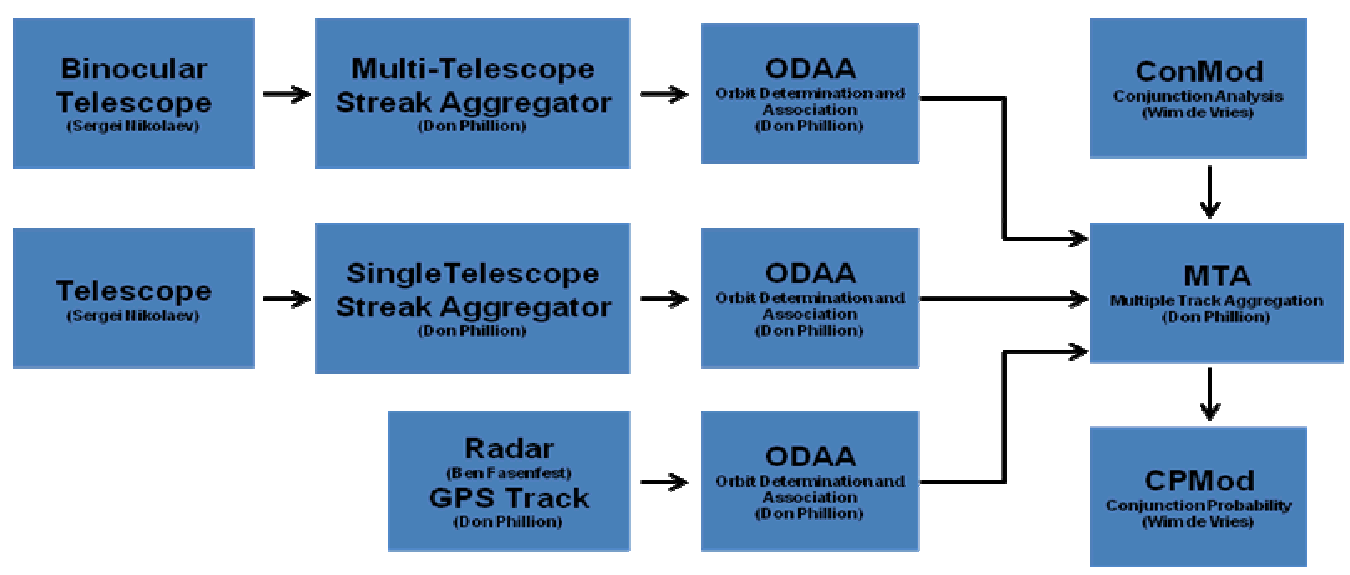

Figure 2. Portion of TESSA architecture that simulates the generation of telescope images, streaks, orbit determination, orbital track aggregation, and performs conjunction analysis and calculation of the collision probability.

\subsection{TESSA simulation framework}

Each of the advanced simulation and modeling techniques described above have been incorporated into the Testbed Environment for SSA (TESSA), a comprehensive SSA enterprise simulation framework, utilizing a parallel discrete event simulation architecture. TESSA is structured as a parallel discrete event simulation (PDES) primarily because the computation is dominated by simulation of the radar and optical sensors at the discrete moments when they make their observations of the sky. It is not, as one might initially surmise, dominated by the continuous computations required for orbital propagation. The simulator is not time-stepped because the simulation times at which the sensor observations occur are not statically predictable, and essentially never coincide. For such models time-stepping would be exceedingly wasteful. An event-driven architecture, which only executes at those simulation times and in those processes where the system state changes, is dramatically more efficient. The TESSA simulator, therefore, is a specially-designed (conservative) distributed discrete event simulation platform. This decision as has important consequences, both for the performance of the TESSA model and for the process of developing it. TESSA has been described previously [2].

\section{RESULTS}

\subsection{Comparison of collision probability behavior for 3 conjunction cases, value of site selection}

Figure 3 compares results for a collision, a near miss ( $100 \mathrm{~m}$ separation), and a far miss $(500 \mathrm{~m})$. The results agree with expectations, where the collision probability increases over time for the collision, rises and then falls for the near miss, and drops precipitously for the far miss. The magenta curves in all three plots are interesting because they use only 3 sites ( 6 telescopes) yet show faster and better orbital refinement that the runs with more telescopes. The difference is that the 3 sites were hand picked to and have good visibility for CFE, with the result that they can take useful observations from an early time. CFE has an orbital inclination of 35.4 degrees, so there is only a $58 \%$ probability that a randomly chosen site will be able to make any observations on CFE because $42 \%$ of the earth's land mass will be at latitudes too high to observe CFE. This shows the premium that exists for a well-located observation site. The statistics of which sites were able to observe CFE agree with the expected 58\%. For example, 7 of 12 sites randomly chosen were able to observe CFE.

The left plot shows that there is little difference in orbital refinement capability for 12 or more sites in the case of a collision. For the near-miss case (center plot), orbital refinement ability improves with increasing number of sites. This is to be expected, since one needs to refine the orbit sufficiently well to discriminate between a collision and a near miss. Interestingly, the hand-picked 3-site case provides comparable results to the randomly-chosen 12 -site case. Given that 
only 7 of the randomly chosen 12 sites are in a location to view CFE at all, and many of those 7 will only be able to make infrequent or poor observations, it is quite reasonable to expect the two cases to produce comparable results.

The right plot shows the expected drop in collision probability after just a few observations for all cases run.
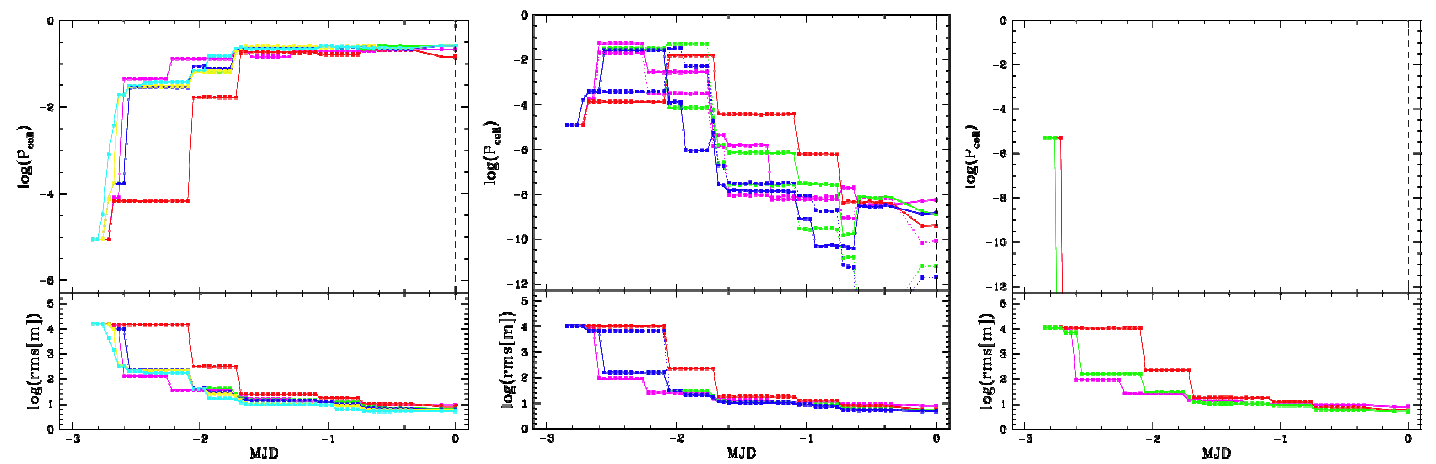

Figure 3. Comparison of a collision (left), near-miss (100 m separation, center), and far miss (500 m, right). The upper panels show the collision probability as a function of Modified Julian Day (MJD) before the collision. The lower panels show the rms value of the error ellipsoid for the satellite position. These are for a (mostly) randomly located set of binocular telescopes, with magenta $=3$ sites ( 6 telescopes), red $=6$ sites, green $=9$ sites, and blue $=$ 12 sites. In the left plot, yellow $=18$ sites, and cyan $=24$ sites. The magenta curves ( 3 sites, 6 telescopes) were manually chosen to have good visibility for the CFE satellite, and consequently show very good orbit refinement capability.

\subsection{Comparison of single and binocular telescopes, and value of more telescopes}

Figure 4 shows simulation results for the case where the two satellites collide, for both a network of single telescopes, left, and a network of binocular telescopes, right. For the single telescopes, the results are essentially identical for 12 or more telescopes. For the binocular telescope, the results are essentially identical for 9 or more binocular sites, which would take 18 telescopes to populate. As in the previous section, the magenta curve for the binocular telescope runs represents three hand-selected ground sites, and might be expected to be comparable to the 12 -site case. The results are very similar for the 3 -site single telescope case and the 3 -site binocular case, which indicates that the number of sites, and not the number of telescopes, is the most important factor for orbital refinement.

Figure 5 shows simulation results for the case where the two satellites have a near miss, for both a network of single telescopes, left, and a network of binocular telescopes, right. For the network of single telescopes, the results are essentially identical for 12 or more telescopes. For the network of binocular telescopes, the best results are obtained for 9 or more sites, with the hand-picked 3 -site case showing the earliest orbital refinement. The data connected with dashed lines in the right plot is for the case where the two orbits intersect, but it is a near-miss temporally. The blue and green dashed data lie to the right of their solid line counterparts, showing that it takes more observations to clarify that this is a near miss and not a collision when the near miss is a temporal one.

\subsection{Comparison of look-ahead times}

The conjunction analysis to screen for potential collisions can be run to an arbitrary number of days in advance, the lookahead time. Earlier warning of a potential collision will have value to the satellite operator, but will mean that the telescope network will be making measurements on more satellites, and that a higher level of orbital refinement will be needed to obtain the same collision/miss discrimination since any errors in the satellite orbit will be propagated further out in time. Here, we have used deterministic orbits, so once an orbit is well-known, that information can be confidently projected in to the future. Future work will include effects such as atmospheric drag and solar pressure which introduce uncertainties to real orbits which results in orbital information becoming less accurate with time unless current observations are made to update the orbit. 

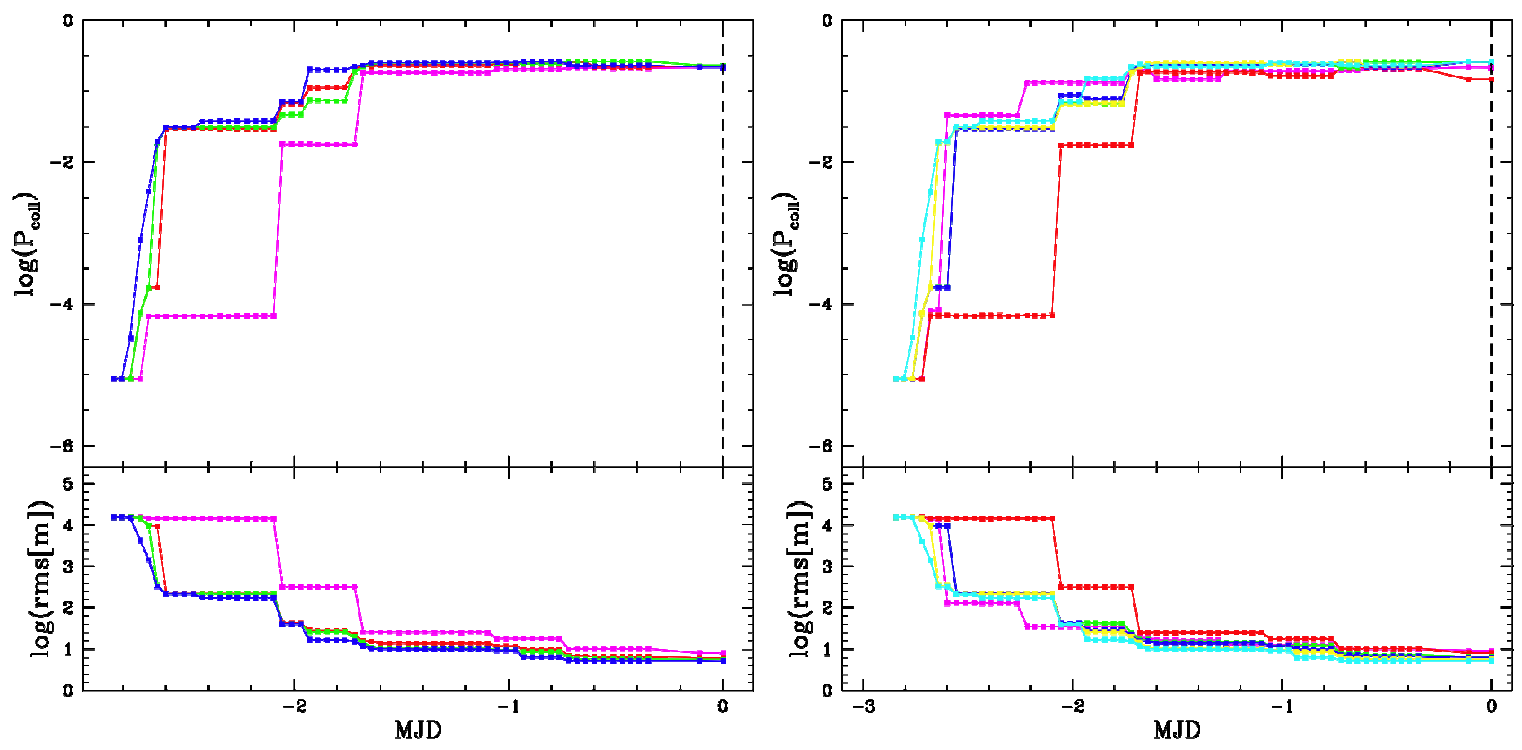

Figure 4. Comparison of collision probabilities for a network of single telescopes (left) and a network of binocular telescopes (right) for the case where the two satellites collide. These are for a (mostly) randomly located set of telescopes, with magenta $=6$ telescopes ( 6 sites left, and 3 sites right), red $=12$ telescopes $(12$ and 6 sites $)$, green $=$ 18 telescopes (18 and 9 sites), and blue $=24$ telescopes ( 24 and 12 sites). In the right plot, yellow $=36$ telescopes (18 sites), and cyan $=48$ telescopes ( 24 sites). The magenta curves ( 3 sites, 6 telescopes) in the right plot were manually chosen to have good visibility for the CFE satellite, and consequently show very good orbit refinement capability.
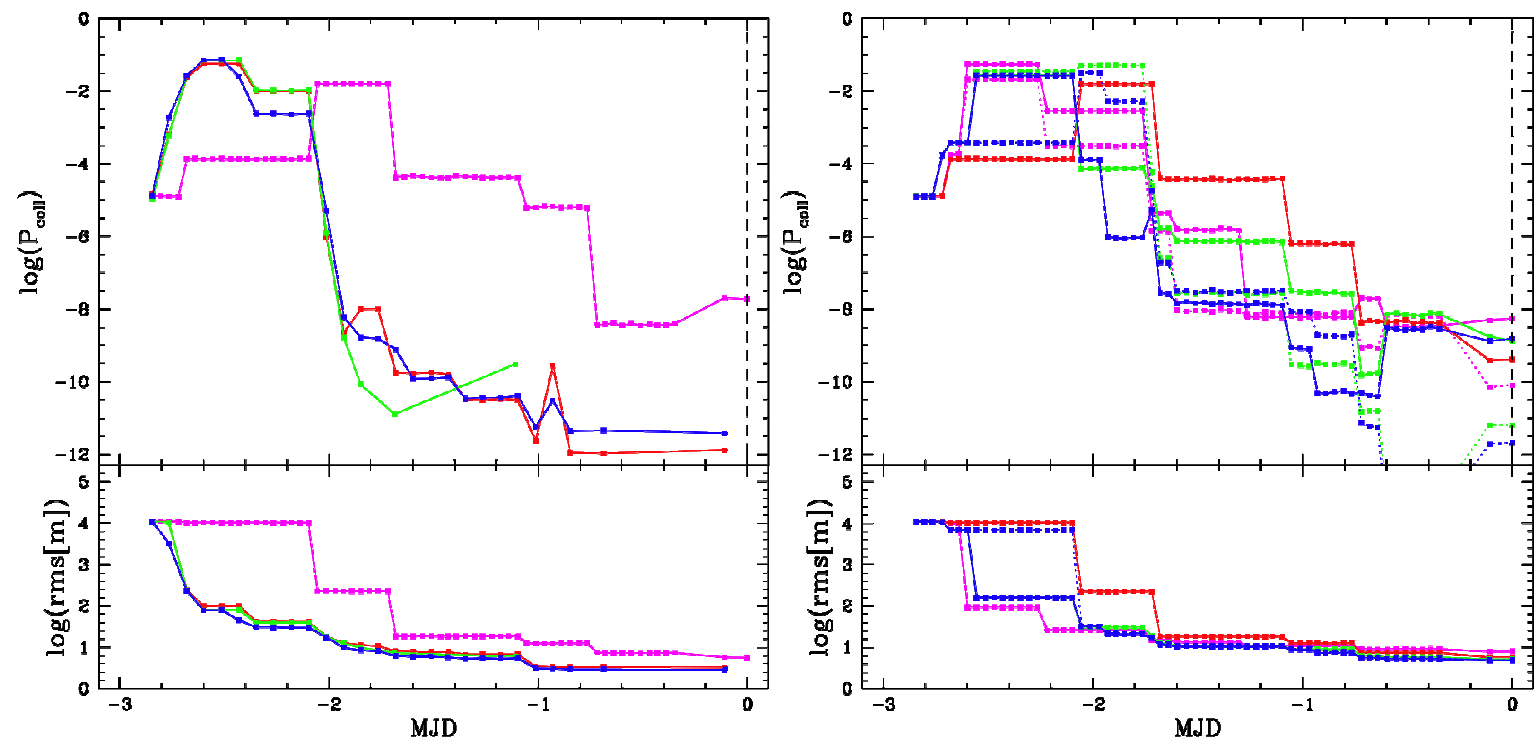

Figure 5. Comparison of collision probabilities for a network of single telescopes (left) and a network of binocular telescopes (right) for the case where there is a near miss. These are for a (mostly) randomly located set of telescopes, with magenta $=6$ telescopes ( 6 sites left, and 3 sites right), red $=12$ telescopes ( 12 and 6 sites), green $=$ 18 telescopes (18 and 9 sites), and blue $=24$ telescopes ( 24 and 12 sites). The magenta curves ( 3 sites, 6 telescopes) in the right plot were manually chosen to have good visibility for the CFE satellite, and consequently show very good orbit refinement capability. The magenta, blue and green data connected with dashed lines in the right plot show the case for a temporal near miss, versus a spatial near miss for all the other plots. 
Figure 6 shows a comparison of a 3-day look-ahead and a 5-day look-ahead for the challenging case of a near miss. In both cases, 18 or more telescopes permit determination that it is a miss (taken to be when $\mathrm{P}_{\text {collision }}<1.0 \mathrm{e}-6$ ) with one day's worth of observations. In the 5-day look-ahead case, it requires 2 days to make the same determination for the 12telescope network, versus 1 day for the 3-day look-ahead. However, that stills gives 3 days notice of the collision, compared to only 2 days notice for the 3 -day look-ahead. For the 6-telescope case, determination that it is a miss takes about 2.5 days for either look-ahead case, but that leaves little time for an avoidance maneuver, if needed, in the 3-day look-ahead.
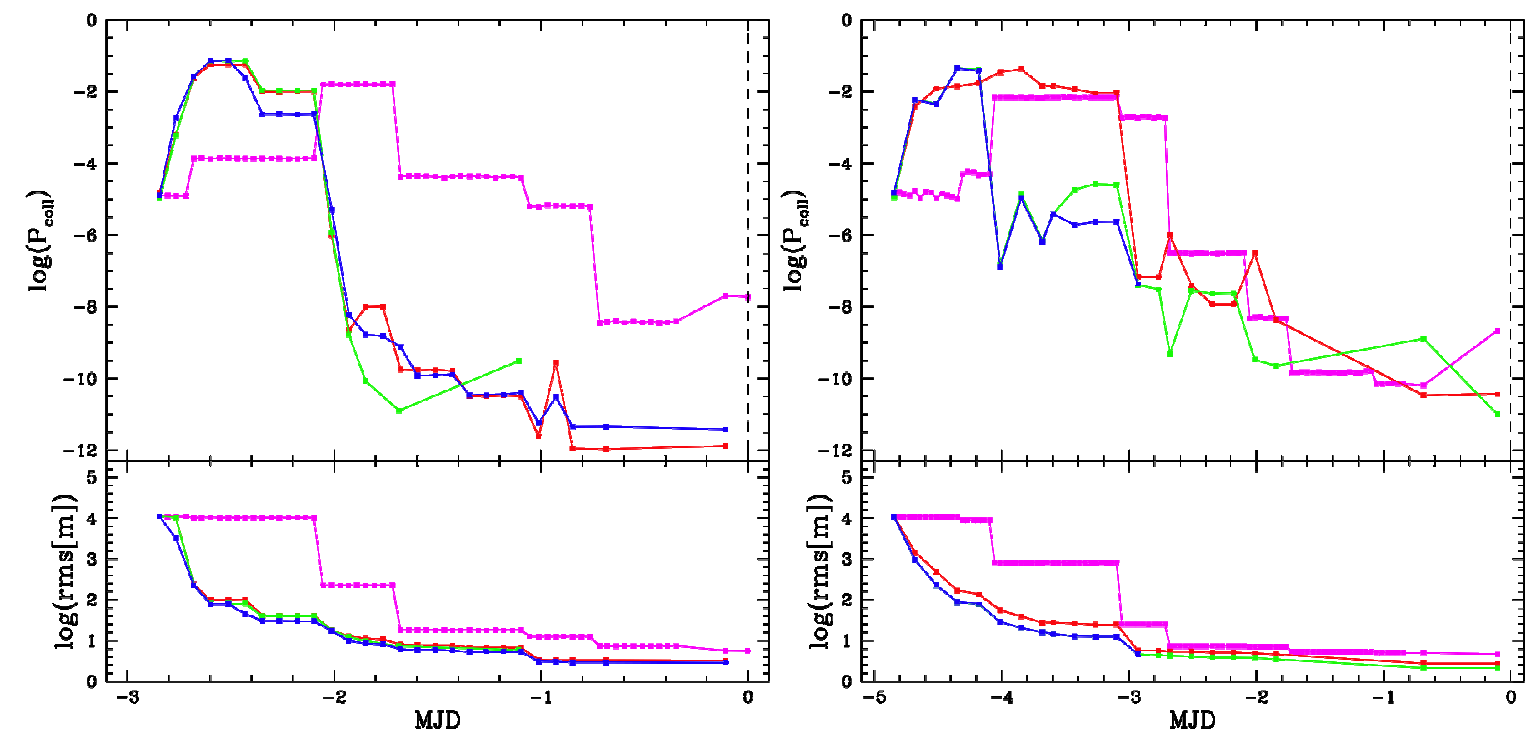

Figure 6. Comparison of a 3-day look-ahead (left) and 5-day look-ahead (right) for the near-miss case. These are for a random set single telescopes, with magenta $=6$ telescopes, red $=12$ telescopes, green $=18$ telescopes, and blue $=24$ telescopes.

\section{ANALYSIS AND CONCLUSIONS}

We examined 3 cases here: (1) a far miss, (2) a collision, and (3) a near miss. A far miss is defined as being the case where the calculated separation at closest approach is greater than the uncertainty in the separation. As discussed in the previous paragraph, the collision probability will go down rapidly as the satellite orbits are refined, because the second satellite is in the tail of the probability distribution. For a collision, the collision probability goes up as the orbits are refined because the probability density at the location of the second satellite increases with the reduction in orbital uncertainty. In the case of a near miss, the behavior is initially like that of a collision, where the calculated collision probability increases with orbital refinement, but eventually the width of the probability distribution comes to be less than the separation between the two satellites, and the collision probability decreases with orbital refinement as described for the far miss case. All three of these behaviors were observed in simulations.

It is important to note that the majority of real-world conjunctions will fall into the first case where additional information continually reduces the probability of collision and it soon becomes apparent that the conjunction will not result in a collision. Our simulations showed that only a few observations (Figure 3, right plot) were required to see a dramatic drop in the collision probability for a far miss.

Discriminating between a collision and a near miss (100 m separation at closest approach) took 1.0 to 1.5 days for most cases, but was typically 1.5 to 2 days for ground networks of 6 telescope sites, but might be as much as 2.5 days if the telescope locations were unfavorable to observe the satellite orbit (Figure 6, right plot). For a collision or a near miss, 9 or more sites were needed to discriminate between a miss and a collision within about a day, although this number is larger with 5-day look-ahead compared to 3-day look-ahead.. 
We also compared distributed networks of single and binocular telescopes, and have started to look at the sensitivity of the results to the locations of the telescopes. We find that a network of $\mathrm{N}$ individual telescopes is best for orbital refinement if an initial orbit is known for an object, but that a network of $\mathrm{N}$ telescopes at N/2 sites in a binocular configuration is best for determining an initial orbit of an object. Careful selection of the location of the telescopes will result in faster and more accurate orbital refinement than random selection of the sites.

Results were compared for both a spatial miss (the two orbits come close, but do not overlap) and a temporal miss (the two orbits overlap, but the satellites do not occupy the overlap region at the same time). As expected, orbital refinement quickly resolves a near-miss in the first case, since the two orbits do not physically overlap. In the case of a temporal miss, more observations are required to discriminate between a collision and a near miss. This is because it is relatively easy to refine the spatial location of an orbit (e.g. $2 \mathrm{~m}$ overlap in an orbit $7 \mathrm{e} 6 \mathrm{~m}$ from the center of the earth, or $0.3 \mathrm{ppm}$ ), but the timing of the orbit requires more observations to get the orbital period to the required accuracy $(0.2 \mathrm{msec}$ out of a 90 minute orbit, or $0.04 \mathrm{ppm}$ ).

\section{FUTURE WORK}

This work has shown that a useful level of orbital refinement can be achieved with a modest number of telescopes. Future work will build on the tools and methodology here to examine trade studies relevant to the implementation of a telescope network for orbital refinement to allow for mitigation of potential orbital collisions. Some of the areas to be pursued are:

- Statistical variability of results. Here, the same random number generator seed was used for all of the reported data. This was done to highlight any trends from increasing the number of telescopes, and avoid statistical fluctuations in the data that might obscure those trends. In general, there is a large number of candidate ground sites, and the orbits of interest are much more varied than the orbit of CFE Sat, which is in LEO and inclined at 53 degrees.

- Optimization of number and location of telescopes. The results of this paper suggest that 12 to 18 single telescopes would be sufficient to quickly refine a satellite orbit. This conclusion needs to be reconsidered for the full constellation of operational satellites. The locations of the ground telescopes were chosen randomly here. It is clear from this work that certain locations often generated a vast improvement in orbit determination, whereas others showed little or no improvement. Optimization of the ground network for the actual configuration of operational satellites would provide a more realistic assessment of the value of the telescope network. Additionally, there is a trade between whether it is preferable to have a given number of telescopes deployed as individual telescopes or as binocular telescopes.

- Other sensors. GPS, ground-based radar, space-based telescopes, and/or IR telescopes may facilitate more rapid orbital refinement, or may provide a more robust system for orbital refinement when the full catalog of operational satellites is considered. TESSA can already handle orbital telescopes. The optical scheduler can generate an observation schedule for observing an orbiting object viewed by an orbiting telescope. Presently only the single telescope streak aggregator can handle orbital streak data, but the multi-telescope streak aggregator with binocular triangulation will soon be modified to also be able to handle orbital data and to do binocular triangulation for orbiting binocular telescopes. The two telescopes comprising an orbiting binocular telescope would be in the same orbit except for having slightly different mean anomalies so as to be separated perhaps by about 20 kilometers. The baseline cannot be too large because the two telescopes comprising the binocular telescope will have the same pointing direction when surveying the sky for new objects in the binocular mode. In order to not lose their projected observational baseline, they would observe well away from the due forward and the due backwards directions for their orbit. A major capability of a binocular telescope is that a rough orbit can be determined from a single observation provided the streak direction is known. If the streak direction is known, then the 3D positions at the streak endpoint times can be determined using binocular triangulation. The midpoint velocity is roughly the difference in the 3D positions divided by the exposure time. The longer the streak, the greater the accuracy. We will study the orbital accuracy that can be achieved both analytically and with TESSA runs. 
- Temporal validity of information. The orbits used here were completely deterministic, which means that once an orbit was well-characterized, that information could be used to predict the satellite position many days ahead. In practice, orbital information more than a few days old has very limited predictive value because there are unknown or inadequately characterized forces, such as atmospheric drag and solar pressure, that alter the orbit over time. There is great operational value in having more advance notice of a likely collision, so understanding the amount of warning time a given system can provide is important.

- Optimized sensor tasking. The work examined only looked at orbital refinement for a single satellite, so there was no competition for observing time at any telescope. In the general case, there may be multiple satellites visible to a given telescope, so an optimized tasking algorithm would be of value. Prime considerations in prioritizing the tasking are the current probability of collision, and the time to potential collision.

- Value of conjunction analysis extending to later times. The three trades here are that more advance notice potentially gives operators more time to perform a collision avoidance maneuver, but that also means more satellites must be tracked, potentially requiring a larger system, and that the early data may have little predictive value, so looking farther ahead may provide only limited additional warning time.

- Quantify risk as a function of separation distance and system configuration. Operators would like to be able to distinguish between a collision and a near-miss, because moving the satellite in the near-miss case will unnecessarily expend limited maneuvering fuel. One can use these tools to generate the Probability of False Alarm (PFA) for a variety of system configurations and near-miss distances. It is expected that the PFA will change with time.

- Capability to acquire orbits of new debris. It took many days after the Cosmos-Iridium collision to detect and catalog the hundreds of pieces of debris generated. These tools can be used to evaluate the ability of different sensor networks to detect and determine orbits for debris generated in a new collision.

\section{ACKNOWLEDGEMENTS}

This work performed under the auspices of the U.S. Department of Energy by Lawrence Livermore National Laboratory under Contract DE-AC51-07NA27344.

\section{REFERENCES}

[1] Col. J. Christopher Moss, "The Joint Space Operations Center and Orbital Debris", presented at the International Conference on Orbital Debris Removal, Chantilly, VA, December 2009.

[2] Olivier, S.S., "A Simulation and Modeling Framework for Space Situational Awareness," Proceedings of the Advanced Maui Optical and Space Surveillance Technologies Conference, Wailea, Maui, Hawaii, 2008.

[3] Canny, J., "A Computational Approach To Edge Detection", IEEE Trans. Pattern Analysis and Machine Intelligence, 8:679-714, 1986.

[4] Fundamentals of Astrodynamics and Applications Third Edition by David A. Vallado. Published jointly by Microcosm Press (Hawthorne CA) and Springer (New York), 2007, pp 457-463.

[5] Fundamentals of Astrodynamics and Applications Third Edition by David A. Vallado. Published jointly by Microcosm Press (Hawthorne CA) and Springer (New York), 2007, pp 431-435. 